\title{
Particle decays during the cosmic dark ages
}

\author{
Xuelei Chen* \\ The Kavli Institute for Theoretical Physics, UCSB, Santa Barbara, California 93106, USA
}

Marc Kamionkowski ${ }^{\dagger}$

Mail Code 130-33, California Institute of Technology, Pasadena, California 91125, USA

(Received 19 October 2003; revised manuscript received 14 April 2004; published 4 August 2004)

\begin{abstract}
We consider particle decays during the cosmic dark ages with two aims: (1) to explain the high optical depth reported by the Wilkinson Microwave Anisotropy Probe (WMAP), and (2) to provide new constraints to the parameter space for decaying particles. We delineate the decay channels in which most of the decay energy ionizes and heats the intergalactic medium gas [and thus affects the cosmic microwave background (CMB)], and those in which most of the energy is carried away-e.g. photons with energies $100 \mathrm{keV} \lesssim E \lesssim 1 \mathrm{TeV}$ - and thus appears as a contribution to diffuse $\mathrm{x}$-ray and gamma-ray backgrounds. The new constraints to the decay-particle parameters from the CMB power spectrum thus complement those from the cosmic x-ray and $\gamma$-ray backgrounds. Although decaying particles can indeed produce an optical depth consistent with that reported by WMAP, in so doing they produce new fluctuations in the CMB temperature and polarization power spectra. For decay lifetimes less than the age of the Universe, the induced power spectra generally violate current constraints, while the power spectra are usually consistent if the lifetime is longer than the age of the Universe.
\end{abstract}

DOI: $10.1103 /$ PhysRevD.70.043502

PACS number(s): 98.80.-k, 95.30.Cq, 95.35.+d, 98.70.Vc

\section{INTRODUCTION}

A large correlation between the temperature and E-type polarization at large angular scale (low $l$ ) was recently observed by the Wilkinson Microwave Anisotropy Probe (WMAP) [1]. This is a unique signature of re-scattering of cosmic microwave background (CMB) photons at redshifts relatively low compared with that of the last-scattering surface at $z \approx 1100$ [2]. The required optical depth of $\tau_{e} \sim 0.17$ can be achieved if reionization occurs at a redshift of $z_{\text {re }}$ $\sim 20$. Although there are theoretical uncertainties, such a reionization redshift is difficult to reconcile with the starformation history expected in the cold dark matter model with a cosmological constant ( $\Lambda \mathrm{CDM}$ model) [3], which generally favors a reionization redshift of 7-12 [4]. Furthermore, the thermal history of the intergalactic medium (IGM) contains further evidence for late completion of reionization [5]. This potential conflict between the evidence for early and late reionization might be partially resolved in the double-reionization model, where an early generation of massive, metal-free stars were formed and partly ionized the Universe [6]. Nevertheless, even in this model, it is not easy to achieve such a high optical depth [1].

In light of this, it is worthwhile to consider possible alternatives. For example, it has been suggested that a high optical depth might be achieved if primordial density fluctuations are non-Gaussian [7]. Here we consider another option. While stellar photons must have contributed to reionization, it remains possible that other energy sources also contribute. Decay of an unstable particle, for example, provides such an alternative energy source. In this scenario, a decaying par-

\footnotetext{
*Electronic address: xuelei@kitp.ucsb.edu

†Electronic address: kamion@tapir.caltech.edu
}

ticle, possibly part of the dark matter, releases energy during its decay, which contributes to the ionization of the IGM. Another widely discussed possibility is the radiative decay of an active neutrino, which might play a role in a number of astrophysical phenomena [8-10]. Although the parameters of the original model are now excluded by observations [11], there are still other regions of decaying-neutrino parameter space, and there is no lack of other particle-physics candidates; e.g., unstable supersymmetric particles [12], cryptons [13], $R$-parity violating gravitinos [14], moduli dark matter [15], superheavy dark-matter particles [16,17], axinos [18], sterile neutrinos [19], weakly interacting massive particles decaying to superweakly interacting massive particles [20], and quintessinos [21]. Recently, Hansen and Haiman [19] suggested sterile-neutrino decay as a source of reionization. In addition to decaying particles, evaporation of primordial black holes [22] and decay of topological defects such as cosmic strings and monopoles are also possible source of extra energy input. The decay of an unstable particle may also help explain the presence of dwarf spheroidal galaxies in the local group, resolve the cuspy halo problem in $\Lambda \mathrm{CDM}$ models [23-25], and serve as a possible source of the ultra high energy cosmic rays [26].

From a cosmological perspective, it is particularly interesting to consider the rich variety of ionization histories offered by the particle-decay scenario. In these scenarios, the Universe is not necessarily fully ionized; instead, particle decay may ionize only a small fraction of the gas. If the process lasts for a large range of redshifts, it may still contribute a large fraction of the measured free-electron optical depth. The presence of a not significantly damped first acoustic peak in the CMB anisotropy spectrum suggests that particle decay should not significantly delay the recombination process at $z \sim 1100[27,28]$, but is it possible that the Universe become partially ionized during the cosmic "dark 
ages" at redshifts of ten to a few hundred? What is the observational signature of such an ionization history? Can this scenario be distinguished from late reionization by CMB observations? Particle decay may also produce energetic photons; can observation of cosmic $\gamma$-ray backgrounds place constraints on this scenario?

In this paper we consider these questions. Since at low redshift stars and quasars emit ionizing photons, and since at the epoch of recombination there is no significant increase of entropy, we shall focus mostly on particle decays in the redshift range between 1000 and 20. Such particles produce an optical depth $\tau \sim 0.17$ by partially reionizing the Universe at redshifts much higher than the value, $z \sim 20$, required if the Universe becomes fully ionized by early star formation. We calculate the CMB temperature and polarization power spectra induced by this alternative ionization history and show that it can be distinguished from the full-reionization scenario with the same $\tau$. In some regions of the decay-particle parameter space, the induced power spectra conflict with those observed already, but there are other regions where decaying particles can provide the required optical depth and maintain consistency with the measured power spectra.

While investigating decaying particles as contributors to cosmic reionization, it becomes clear that new CMB constraints to the ionization history provide new constraints to the parameter space for decaying particles. To a first approximation, the energy injected by particles that decay with lifetimes between the ages of the Universe at recombination and today either gets absorbed by the IGM, or it appears in diffuse radiation backgrounds [29]. In the latter case, observed radiation backgrounds have traditionally been used to constrain the parameter space that consists of the decay-particle lifetime and density as well as the energy of the decay products. As we detail below, new CMB constraints to the ionization history can now provide complementary new constraints to the regions of parameter space where the decay energy goes to heating and ionizing the IGM.

This paper is organized as follows: In the next section, we discuss how energy is dissipated for various decay channels and what fraction of energy is eventually used for ionization, heating the gas, or carried away by escaping photons and neutrino. We also discuss how this energy is deposited as a function of redshift. In Sec. III, we describe how to calculate the ionization history and CMB anisotropy with extra energy input from decaying particles, and we discuss how the result depends on the property of the particle. In Sec. IV, we obtain constraints to the decay-particle parameter space from the $\mathrm{CMB}$ and diffuse backgrounds. We summarize our results in Sec. V. ${ }^{1}$

\section{DECAYING CHANNELS AND ENERGY DISSIPATION}

Depending on the nature of the decaying particle, the decay products may include gauge bosons, charged leptons,

\footnotetext{
${ }^{1}$ While this paper was being prepared, two papers [30,31] on similar questions appeared. Our results agree with theirs where our calculations overlap.
}

neutrinos, quarks, or other more exotic particles. These particles may then subsequently decay further into other particles, or they may interact with particles in the IGM. With sufficient energy, a shower of particles is created. In the end, stable, weakly interacting particles like neutrinos escape, while other particles lose a significant part of their energy during the interaction with the primordial gas or cosmic microwave background. Some of this energy can go into ionizing the IGM, and the efficiency of converting the decay energy to ionization energy is process dependent. Here we review some of the more generic features; in particular, we consider the efficiency of converting the rest mass of the decaying particle to ionization energy, $\chi_{i} \equiv E_{i} / M_{X} c^{2}$, where $M_{x}$ is the mass of the decaying particle.

\section{A. Photons}

In this paper we are mostly interested in the "dark ages," $10<z<1000$, where most of the gas is neutral. Photons with energy smaller than $13.6 \mathrm{eV}$ cannot ionize hydrogen atoms in the ground state, but if there is a large presence of hydrogen atoms in excited states, e.g. at the end of the recombination era, $z \sim 1000$, photons with energy $E<13.6 \mathrm{eV}$ may contribute to the ionization. When most of the atoms fall to the ground state, photons with energy less than $13.6 \mathrm{eV}$ will escape.

Ultraviolet and soft $\mathrm{x}$-ray photons with energy $13.6 \mathrm{eV}-1 \mathrm{keV}$ have large photoionization cross sections and are largely absorbed locally. For photons with energy $E>40 \mathrm{eV}$, neutral helium absorption dominates the absorption, and photoelectrons are produced in the process. The photoelectron carries the remaining energy of the photon. From here on the energy dissipation process for the initial photon is the same as that for an initial energetic electron.

The absorption processes of hard $\mathrm{x}$-ray and $\gamma$-ray photons at cosmological distances were discussed in Ref. [32]. The processes in which photons can be absorbed or lose energy include (i) photoionization of atoms, (ii) Compton scattering on electrons, (iii) production of pairs on atoms, (iv) production of pairs on free electrons and free nuclei, (v) scattering with background photons, and (vi) pair production on background photons. In some of these, such as Compton scattering, a photon loses only a small fraction of its energy in a single scattering event, while in others it can lose a significant part of its energy.

In Fig. 1, we plot the total energy-loss rate,

$$
-\frac{d \ln E}{d \ln (1+z)}=\frac{\Delta E}{E} \frac{n(z) \sigma(E) c}{H(z)},
$$

as a function of energy for redshifts, $1+z=10,100$, and 316 . We assume $\Delta E / E \sim 1$ except for the Compton-scattering process. Here, $H(z)$ is the expansion rate of the Universe at redshift $z, n(z)$ is the density of the target particle-i.e., neutral hydrogen or helium for (i) or (iii), free electrons for (ii) or (iv), and CMB photons for (v) or (vi) - and $\sigma$ is the corresponding cross section. At the high energy ( $E$ $\ll 13.6 \mathrm{eV}$ ) where Compton scattering becomes important, a photon is not able to distinguish whether an electron is free 


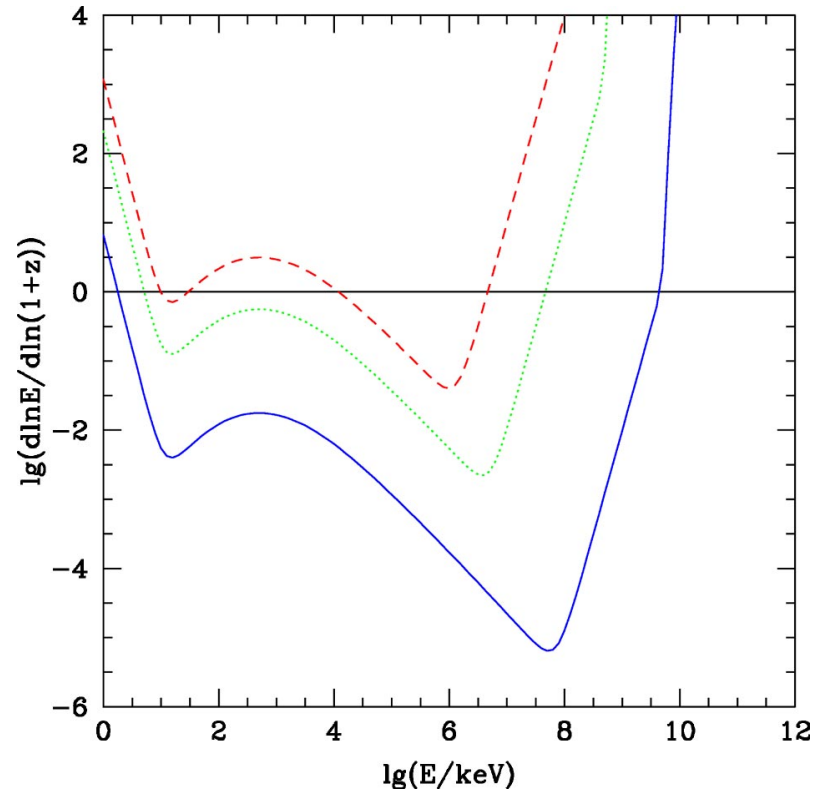

FIG. 1. Energy loss rate by photons per Hubble time. The solid (blue), dotted (green), and dashed (red) lines are for redshift $z+1$ $=10,100$, and 316, respectively. We took $x_{e}=0.01$ here, but the results are insensitive to $x_{e}$ for $E>1 \mathrm{keV}$.

or bound, ${ }^{2}$ so we assume a free-electron fraction of 0.01 for processes (i), (iii), (iv), but treat all electrons as free for the Compton processes. Our result is not sensitive to the ionization fraction as long as the Universe is mostly neutral. We should also point out that in this discussion we have also neglected any other photon background, e.g. the infrared photon background which might be produced at low redshift.

As we can see from the figure, high-energy photons (above $1 \mathrm{TeV}$ at $z \sim 10,1 \mathrm{GeV}$ at $z \sim 300$ ) can scatter with $\mathrm{CMB}$ photons or produce pairs, and they are largely absorbed locally, producing either an x-ray photon which has a larger absorption cross section, or an electron-positron pair with high energy.

For photons with energy around $1 \mathrm{GeV}$ (and around $1 \mathrm{keV}$ at low redshift), the Universe is optically thin in the redshift range which we are interested in, photons lose most of their energy by redshifting, only a small fraction of the total energy is transferred to gas by scattering, and the scattering occurs over a wide range of redshifts. The details of the energy distribution depend on the injection energy and redshift of the photon. The formation of an electromagnetic shower and the resulting spectrum was investigated for both low redshift $[17,34,35]$ and the early Universe $[36,37]$. The spectrum of the shower is of the form $E^{-\alpha}$ below the threshold energy, with $0<\alpha<2$ [38].

Figure 2 shows the transparency window. In the dark regions, $d \log E / d \log (1+z)>1$ (i.e., most of the photon energy goes to the IGM), and in the white regions $d \log E / d \log (1$ $+z)<1$ (i.e., the Universe is transparent to these photons). The bump in the transparency window at $(\log E, \log (1+z))$

\footnotetext{
${ }^{2}$ This was pointed out to us by Professor R. Sunyaev; see e.g. [33] for more detailed discussion.
}

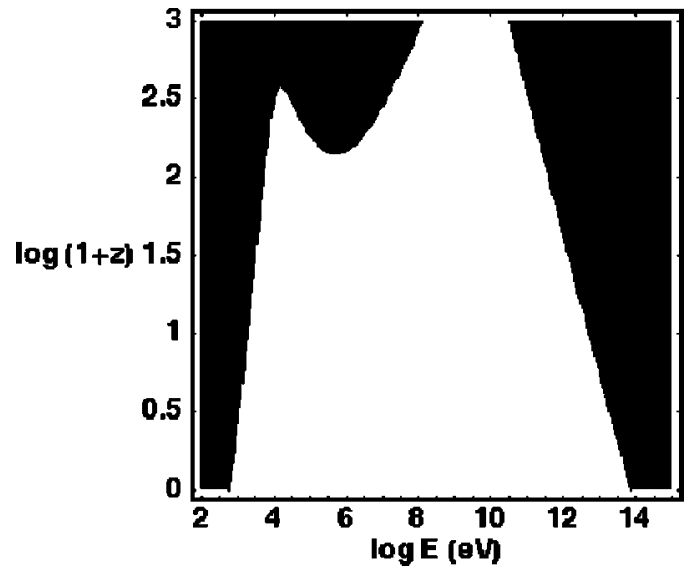

FIG. 2. Transparency window for photons. The dark regions are those in which $d \log E / d \log (1+z)>1$; i.e., those in which most of the photon energy gets absorbed by the IGM in a Hubble time. In the clear regions, the Universe is transparent to photons.

$=(5.7,2.16)$ is due to the Compton scattering. If the photon is injected in the transparency window, and remains in the transparency window as its energy redshifts (once a photon is injected it travels down and to the left on this plot), then it will free stream and appear in diffuse radiation backgrounds with energies $\sim \mathrm{keV}-10 \mathrm{TeV}$; otherwise, it will not appear as a diffuse background, but will heat and ionize the IGM. Also keep in mind that the time interval $d t \propto d z(1+z)^{-5 / 2}$. Thus, if a particle decays with lifetime longer than the age of the Universe, the relevant redshift for determining whether it appears in diffuse backgrounds is $z=0$. In Sec. IV below, we will give constraints to the decay-particle parameter space under two extreme assumptions: (1) that all the photon energy goes into the IGM, and (2) that the photons free stream and appear as diffuse backgrounds. Figure 2 must then be consulted to determine which limit applies for a particular decay-photon energy and lifetime.

What we need to know for the ionization history is what fraction of the energy is converted to ionization energy, and how it is distributed over redshift. In the optically thick case-i.e. for photons outside the transparency window-we can assume that the energy is instantly deposited. The energy deposition rate (in units of $\mathrm{erg} \mathrm{cm}^{-3} \mathrm{~s}^{-1}$ ) is

$$
Q(z)=\chi_{i}(z) n_{X}(z) M_{X} c^{2} \Gamma_{X},
$$

where $M_{X}$ is the mass of the decaying particle, and $\Gamma_{X}$ the decay rate. If the lifetime of the particle is much longer than the age of the Universe and $\chi$ is constant, then $Q(z) \propto(1$ $+z)^{3}$.

In the optically thin case, the efficiency is much lower. Local absorption is negligible, and a flux of high-energy photons is produced. These photons may interact with baryons by photoionization, Compton scattering, and pair creation, and with cosmic-radiation-background photons by photonphoton scattering. After interaction, the energy is transferred to the electrons, positrons, and ions which have stronger interactions with other particles, or to photons with much smaller energy and greater optical depth. Here we make the 
following approximation: Once a scattering happens at redshift $z$, the energy carried by the photon at that redshift is completely deposited with some efficiency. This seems to be a good approximation, since the electrons and lower-energy photons produced in the scattering event have much greater optical depths. The energy deposition rate is then

$$
\begin{aligned}
Q(z)= & 4 \pi \int d E\left[\chi_{b} n_{b}(z) \sigma_{b \gamma}(E)\right. \\
& \left.+\chi_{\gamma} n_{\gamma}(z) \sigma_{\gamma \gamma}(E, z)\right] F(E, z)
\end{aligned}
$$

where $\chi_{b}, \chi_{\gamma}$ are efficiencies for converting the energy of that photon to ionization energy, and $\sigma_{b \gamma}(E)$ and $\sigma_{\gamma \gamma}(E, z)$ are the cross sections for interacting with baryons and background photons, respectively, the latter also depending on $z$ since the background photon energy changes. In the approximation of low optical depth (neglecting absorption), the flux is given by

$$
F(E, z)=\frac{c}{4 \pi} \int_{z}^{\infty} \frac{d z^{\prime}}{\left(1+z^{\prime}\right) H\left(z^{\prime}\right)} \frac{J\left[\left(1+z^{\prime}\right) /(1+z) E, z^{\prime}\right]}{\left(1+z^{\prime}\right)^{3} /(1+z)^{3}},
$$

where $J(E, z)$ is the emissivity at $z$. For simplicity, let us consider the case where the decay process directly produces photons with a single energy $E_{\gamma}=x M_{X} c^{2}$. For example, if the decay products are two photons, $x=1 / 2$. In other cases, the photon may not have a single energy, but still it is expected the energy of the photon is related to the mass of the decaying particle and has a narrow range. Then $J(E, z)$ $=N_{\gamma} M_{X} c^{2} n_{X}(z) \Gamma_{X} \delta\left(E-x M c^{2}\right)$, where $N_{\gamma}$ is the number of photon emitted in a decay, and the flux is given by

$$
F(E, z)=\left.\frac{c}{4 \pi}\left(\frac{E}{x M_{X} c^{2}}\right)^{3} \frac{N_{\gamma} \Gamma_{X} n_{X}\left(z^{\prime}\right)}{H\left(z^{\prime}\right)}\right|_{\left(1+z^{\prime}\right) /(1+z)=x M_{X} c^{2 / E}} .
$$

Integrating over $E$, if the interactions with baryons dominate, then

$$
Q(z) \sim \frac{c n_{b}(z) \sigma_{\mathrm{eff}}}{H(z)} Q_{0}(z)
$$

where $Q_{0}(z)$ is the expression given in Eq. (2) with $\chi_{i}$ $=N_{\gamma} \chi_{b}$,

$$
\sigma_{\mathrm{eff}}=\int \frac{d E}{x M c^{2}} \sigma_{b \gamma}(E)\left(\frac{E}{x M c^{2}}\right)^{3 / 2} .
$$

The efficiency is roughly suppressed by a factor of $\tau_{S}$ $\sim n_{b}(z) c \sigma / H(z)$, i.e. the optical depth for the Hubble length. If interaction with background radiation photons dominate, the suppression factor is $\tau_{S}$ $\sim n_{\mathrm{CMB}}(z) c \sigma(z) / H(z)$. The redshift dependence is stronger due to the additional factor of $1+z$ in the cross section.

\section{B. Electrons}

An electron can collide with and ionize atoms, or it can inverse-Compton scatter CMB photons, a process that produces an energetic photon. Those photons will be absorbed again, starting an electromagnetic shower. The energy loss $d E$ of an electron per unit distance $d x$ by ionization in a neutral hydrogen gas is given by [39]

$$
\begin{aligned}
-\frac{d E}{d x}= & \frac{2 \pi e^{4} n_{H}}{m v^{2}}\left[\ln \frac{\left(m v^{2} \gamma^{2}-T_{m}\right)}{2 I^{2}}+\frac{1}{\gamma^{2}}-\left(\frac{2}{\gamma}-\frac{1}{\gamma^{2}}\right) \ln 2\right. \\
& \left.+\frac{1}{8}\left(1-\frac{1}{\gamma}\right)^{2}\right]
\end{aligned}
$$

where

$$
T_{m}=\frac{2 \gamma^{2} m_{H}^{2} m_{e} v^{2}}{m_{e}^{2}+m_{H}^{2}+2 \gamma m_{e} m_{H}} .
$$

The energy loss by inverse Compton scattering is given by

$$
-\frac{d E}{d t}=\frac{4}{3} \sigma_{T} c U_{\mathrm{CMB}} \gamma^{2} .
$$

Other forms of energy loss are relatively unimportant for reasonable values of parameters. For example, synchrotronradiation loss is

$$
-\frac{1}{E} \frac{d E}{d t}=1.05 \times 10^{-31} \frac{E}{\mathrm{MeV}}\left(\frac{B}{\mu \mathrm{G}_{\mathrm{s}}}\right)^{2} .
$$

Since the energy-loss rate for inverse-Compton scattering is proportional to $\gamma^{2}$, it dominates at high energy. At low energy, the ionization-loss rate is given approximately by

$$
-\frac{d E}{d x} \approx 2.54 \times 10^{-19} n_{H}(3 \ln \gamma+20.2) \mathrm{eV} / \mathrm{cm} .
$$

Since $n_{H} \propto(1+z)^{3}$ and $U_{\mathrm{CMB}} \propto(1+z)^{4}$, inverse-Compton scattering dominates the energy loss at

$$
z \gtrsim 20.8\left(\frac{\Omega_{b} h^{2}}{0.022}\right)\left(\frac{2.726}{T_{0}}\right)^{4} \gamma^{-2} .
$$

We plot the energy-loss rate as a function of $E$ for $z=0$ and $z=20$ in Fig. 3. Generally speaking, if the electron has energy greater than $\sim 100 \mathrm{eV}$ but smaller than $\sim \mathrm{MeV}$, the energy-loss mechanisms are collisional ionization and excitation. If the electron has energy greater than $\sim \mathrm{MeV}$, it loses most of its energy by inverse-Compton scattering, producing $\mathrm{UV}$ and x-ray photons. If the electron energy is $E_{e} \lesssim \mathrm{GeV}$ or $E_{e} \gtrsim 50 \mathrm{TeV}$, these photons are subsequently absorbed by photoionization and excitation (or by pair production), and the decay energy is thus transferred locally to the IGM. However, if the electron has an energy $1 \mathrm{GeV} \leq E_{e}$ $\lesssim 50 \mathrm{TeV}$, the up-scattered $\mathrm{CMB}$ photon has an energy in the transparency window $10 \mathrm{keV}-10 \mathrm{TeV}$. Thus, if the injected electron has an energy in this "electron transparency 


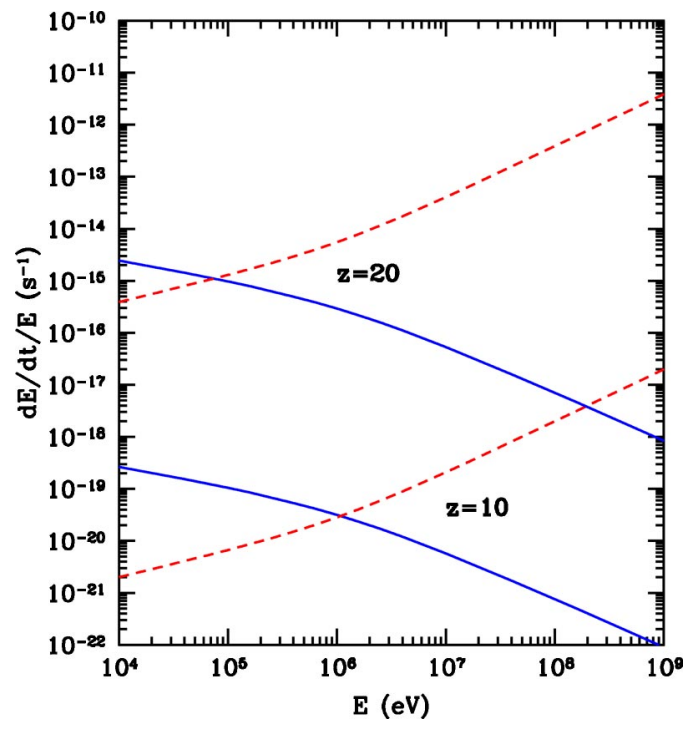

FIG. 3. Energy loss rate due to ionization (blue solid line) and inverse Compton scattering (red dashed line) of an energetic electron. We plot for the cases of $z=10$ and $z=20$.

window," the decay energy will escape and appear in diffuse photon backgrounds, and will not transfer most of its energy to the IGM.

For the case where the electron does heat the IGM, the partition of the energy among ionization, excitation, and heating was investigated by Shull and Van Steenberg [40]. They found that when the gas is mostly neutral, about $1 / 3$ of the energy goes to ionization, about the same amount goes into excitation, and the rest heats the IGM. For a fully ionized medium, almost all of the energy goes into heating the gas. Therefore, we can approximate the fraction of energy going into ionization as

$$
\chi_{i} \sim \chi_{e} \sim\left(1-x_{e}\right) / 3, \quad \chi_{h} \sim\left(1+2 x_{e}\right) / 3 .
$$

This approximation is crude but sufficiently accurate for our purposes.

If the energy of the initial electron is still higher, $E$ $\sim 1 \mathrm{MeV}^{2} / E_{\mathrm{CMB}} \sim 10^{10}(1+z)^{-1} \mathrm{MeV}$, it can scatter with another photon or electron and produce an electron-positron pair. The electron and positron then lose energy through inverse-Compton scattering or ionization, the positron eventually annihilates with another electron and produces $511 \mathrm{keV}$ photons. To summarize, roughly one-third the decay energy goes locally into ionization, and the rest into heating the gas, unless the electron is injected in the transparency window $\mathrm{GeV} \lesssim E \lesssim 50 \mathrm{TeV}$, in which case most of the energy is carried away by upscattered CMB photons.

\section{Other particles}

Protons are very penetrating particles and thus are not effective in transferring decay energy to the IGM.

Other particles. Other decay products (e.g., muons, tau leptons, heavy quarks, gauge, or Higgs bosons) will generally produce showers of lower-energy particles. This is a complicated and model-dependent process. In the absence of a given well-motivated candidate that decays to these particles, we neglect to carry out a detailed analysis. Roughly speaking, we expect typically $10 \%$ of the decay to wind up in ionization energy at the decay redshift, with a comparable amount going to heating the gas, and the rest begin carried away by neutrinos or as rest-mass energy of decay particles.

\section{IONIZATION HISTORY}

The decay of an unstable particle can affect both recombination at $z \sim 1000$ and reionization at low $z$, or it may peak at a middle redshift. However, since the first acoustic peak is not significantly damped, recombination must be rapid, and completed well before $z \sim 100$, when the Universe starts to become optically thin even at full ionization [27].

In the presence of the decaying particle, the evolution of the ionization fraction $x_{e}$ satisfies

$$
\frac{d x_{e}}{d z}=\frac{1}{(1+z) H(z)}\left[R_{s}(z)-I_{s}(z)-I_{X}(z)\right],
$$

where $R_{s}$ is the standard recombination rate, $I_{s}$ the ionization rate by standard sources, and $I_{X}$ the ionization rate due to particle decay. This last term is related to the energydeposition rate $Q$ introduced earlier: $I_{X}=Q(z) / n_{b}(z) / E_{0}$, where $E_{0}$ is the average ionization energy per baryon. At low redshift, the standard ionization sources are photons from stars or active galactic nuclei (AGN), $I_{s}=I_{*}$, and the standard recombination rate is

$$
R_{s}=C_{\mathrm{HII}} \alpha_{B}(T) x_{e}^{2} n_{b}(z)
$$

where $\alpha_{B}(T)$ is the case B recombination coefficient for gas at temperature $T$ and density $n_{b}$. Here $C_{\mathrm{HII}}$ is the clumping factor. We take $C_{\mathrm{HII}}=1$, appropriate for $z \gtrsim 20$ [41].

The number density of decaying particles is proportional to $(1+z)^{3} e^{-\Gamma_{X} t}$, and its energy density is simply the number density times the rest mass. The particle-decay ionization rate is

$$
I_{X i}=\epsilon_{X i}(z) H \text { with } \epsilon_{X i}(z)=\chi_{i}(z) \frac{M_{X}}{E_{0}} \frac{n_{X}(z)}{n_{b}(z)} .
$$

To simplify the analysis, we neglect the effect of helium and assume $m_{b}=m_{H}$ and $E_{0}=13.6$. The partition of ionization energy in hydrogen and helium depends on the nature of the decaying particle. The helium atom has a greater ionization energy and also a greater photonionization cross section, so it will probably take away more energy than hydrogen and produce fewer electrons. However, it should not affect the order of magnitude of our estimate. The ratio $m_{b} / E_{0} \sim 7 \times 10^{7}$; thus even only a tiny number of particle decays may supply enough energy to reionize the Universe.

Since there are no stars present at recombination, $\mathrm{CMB}$ photons are the main source of ionization. In this case, a recombination to the ground state produces an ionizing photon which immediately ionizes another atom and thus produces no net change in the ionization fraction; only recombination to the $n \geqslant 2$ state produce net recombination. 
Assuming the number of photons in the $2 s$ state given by the thermal-equilibrium value, the net recombination rate is

$$
R_{s}-I_{s}=C\left[\alpha_{B}(T) x_{e}^{2} n_{b}(z)-\beta_{T}\left(1-x_{e}\right) e^{-E_{2 s} / k T_{M}}\right],
$$

where $\beta_{T}$ is the photoionization coefficient,

$$
C=\frac{1+K \Lambda n_{b}\left(1-x_{e}\right)}{1+K(\Lambda+\beta) n_{b}\left(1-x_{e}\right)},
$$

and $\Lambda=8.23 \mathrm{~s}^{-1}$ is the two-photon decay rate of the $2 \mathrm{~s}$ level. During this epoch, particle decay increases the ionization rate not only by direct ionization from the ground state, but also by contributing additional Lyman-alpha photons which boost the population at $n=2$, increasing the rate of photoionization by the CMB from these excited states,

$$
I_{X}(z)=I_{X i}(z)+I_{X \alpha}(z),
$$

where $I_{X i}$ is the ionization rate given above, and $I_{X \alpha}$ the ionization rate due to additional Lyman alpha photons,

$$
I_{X \alpha}=(C-1) \epsilon_{\alpha}(z) H, \quad \epsilon_{X \alpha}(z)=\chi_{\alpha}(z) \frac{M_{X}}{E_{\alpha}} \frac{n_{X}(z)}{n_{b}(z)} \frac{\Gamma_{X}}{H} .
$$

Using $n_{X}=\Omega_{X} \rho_{c} / M_{X}$ and $n_{b}=\Omega_{b} \rho_{c} / m_{b}$, where $\Omega_{X}(z)$ is the fractional abundance of the decaying particle at $z, M_{X}$ the mass of the decaying particle, and $m_{b}$ the mean baryon mass, we have

$$
I_{X i}(z)=\chi_{i}(z) \frac{m_{b}}{E_{0}} f_{X} \Gamma_{X}, \quad I_{X \alpha}(z)=\chi_{\alpha}(z) \frac{m_{b}}{E_{\alpha}} f_{X}(z) \Gamma_{X},
$$

where $f_{X}=\Omega_{X}(z) / \Omega_{b}(z)$. Written in this form, the ionization rate depends only on the fractional abundance of the particle and the ionization efficiency. The gas-temperature evolution is given by

$$
\begin{aligned}
(1+z) \frac{d T_{b}}{d z=} & \frac{8 \sigma_{T} a_{R} T_{\mathrm{CMB}}^{4}}{3 m_{e} c H(z)} \frac{x_{e}}{1+f_{\mathrm{He}}+x_{e}}\left(T_{b}-T_{\mathrm{CMB}}\right) \\
& -\frac{2}{3 k_{B} H(z)} \frac{K(x)}{1+f_{\mathrm{He}}+x_{e}}+2 T_{b},
\end{aligned}
$$

where

$$
K(x)=\chi_{h} m_{b} \frac{\Omega_{X}(z)}{\Omega_{b}(z)} \Gamma_{x} .
$$

We use a modified version of the code RECFAST [42] to calculate these rates and derive the ionization history of the gas for a given decaying particle.

Once the ionization history is obtained, the CMB anisotropy can be calculated by modifying a standard Boltzmann code. We have used CAMB [43] for our calculation. Except for the power-spectrum normalization and reionization optical depth, we adopt the WMAP best-fit parameters for the

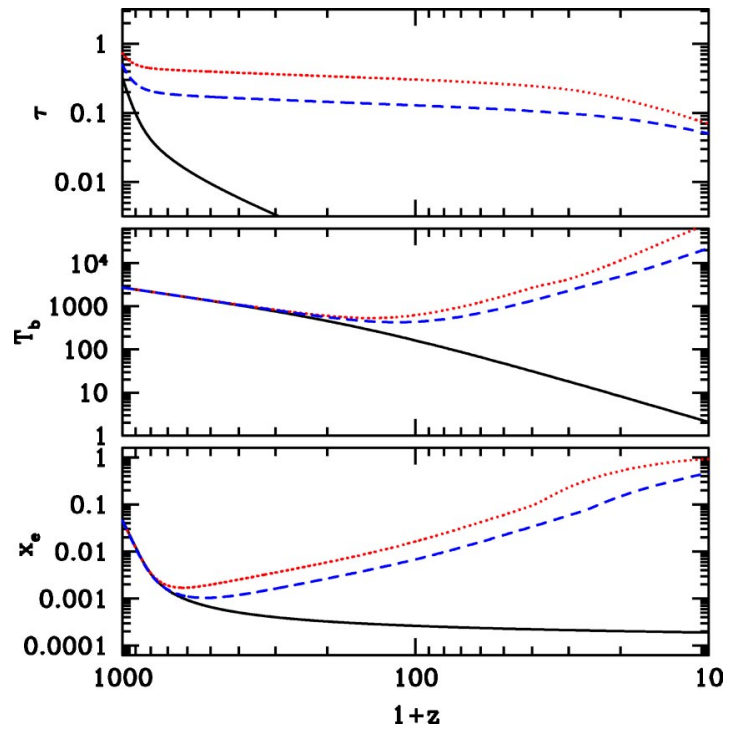

FIG. 4. The optical depth, IGM temperature, and ionization fraction as a function of redshift for standard recombination with no reionization (black solid line) and a decaying-particle model with two-particle decay with $\Gamma_{X} \ll H_{0}$ and $\xi \equiv \chi_{i} f_{X} \Gamma=2.4 \times 10^{-23} \mathrm{~s}^{-1}, \tau$ $=0.4$ (red dotted line), and $0.6 \times 10^{-23} \mathrm{~s}^{-1}, \tau=0.17$ (blue dashed line).

flat $\Lambda$ CDM model with a power-law spectrum [1]; i.e. $\left\{\Omega_{m 0} h^{2}, \Omega_{b 0} h^{2}, h, n_{s}\right\}=\{0.14,0.024,0.72,0.99\}$.

\section{A. Decaying particle with long lifetime}

The decaying particle may or may not be a major component of the dark matter. If $\Gamma \ll H_{0}$, and the primary decaying particle is massive, then $\Omega_{x}(z) / \Omega_{b}(z)$ is effectively constant, and the ionization history depends only on the energy output $\xi=\chi_{i} f_{x} \Gamma_{x}$. After recombination, the ionization fraction induced by the decaying particles may be estimated from the Saha equation as $^{3}$

$$
x_{e}=\left(\epsilon_{i} H / \alpha_{B} n_{b}\right)^{1 / 2} \propto(1+z)^{-3 / 2} .
$$

As the Universe expands, the physical density drops, the recombination rate decreases, and the ionization fraction increases, until at a certain point the Universe is fully ionized, or ionization by decaying particles is exceeded by stellar reionization. The contribution to the optical depth is then

$$
\tau=\int \frac{c d z}{H} \sigma_{T} x_{e}(z) n_{b 0}(1+z)^{2} \propto \ln (1+z) .
$$

In Fig. 4 we plot the ionization history for the case of a long-lived decaying particle $\left(\Gamma_{X} \ll H_{0}\right)$ and instant energy deposition, but with different energy output $\xi$. In this figure, $\tau$ is the Thomson optical depth between today and redshift $z$; $T_{b}$ is the gas temperature; and $x_{e}$ is the ionization fraction. At

\footnotetext{
${ }^{3}$ If there is no extra ionization, the Saha equation does not necessarily describe the ionization state as ionization reactions may have already frozen out because of the paucity of free electrons.
} 
$z \sim 1000$ the ionization fraction drops rapidly due to rapid recombination and to the decrease of the ionization rate by the CMB. When particle decay starts to dominate the ionization rate at $z \sim 600-800$, the ionization fraction starts to increase again because now the ionization rate is constant while the recombination rate drops. At $z \sim 100$, the ionization fraction can reach a few percent, two orders of magnitude higher than in standard models. The optical depth increases slowly with $z$.

The temperature of the IGM also starts to increase at $z$ $\sim 100$, and continues to climb as $z$ decreases. The reason for this is that as the neutral fraction decreases, with the efficiency assumed in Eq. (14), more and more energy is converted to heat at low redshift, and also at lower redshift the baryons and $\mathrm{CMB}$ photons are kinetically decoupled. The difference in the CMB and gas temperature produces distortions to the CMB blackbody spectrum, which are quantified by the Compton- $y$ parameter,

$$
y=\sigma_{T} c \int \frac{k_{B}\left(T_{e}-T_{\mathrm{CMB}}\right)}{m_{e} c^{2}} \frac{x_{e} n_{b}(z) d z}{(1+z) H(z)} .
$$

However, for this and all other models studied in this paper, we found this effect induces $y<10^{-8}$, well below the current limit [45].

How does the additional ionization by particle decay affect the CMB anisotropy? As is well known (see [2] and references therein), for the temperature anisotropy there is only a weak effect on large scale, but on small scales the temperature spectrum is damped by a factor of $e^{-2 \tau}$. The division of large and small scale is determined by the angular size of the horizon at the reionization redshift. In principle, the power-spectrum normalization can also be determined by other measurements [46]. However, other parameters also affect small-scale anisotropy. To avoid re-fitting all the cosmological parameters, we simply fix all other parameters, and adjust the overall normalization to fit the WMAP TT and TE data. ${ }^{4}$

So far we have considered only energy input from particle decay. At low redshifts, stars and quasars contribute a large part (if not all) of the ionizing photons. Since it is not the aim of this paper to provide a detailed model of the starformation history, we simply illustrate the effects of particle decay on the $\mathrm{CMB}$ power spectrum by using an ionizing flux due only to particle decay for $z>z_{*}=7$; we then assume the Universe became suddenly and permanently reionized by standard sources below that redshift. We then calculate the CMB temperature and polarization anisotropy for this ionization history.

The renormalized $\mathrm{CMB}$ temperature and polarization power spectra are plotted in Fig. 5 (all $l$ 's) and Fig. 6 (low $l$ 's). There is practically no difference in the TT spectrum for the different models at high $l$, although there are small dif-

\footnotetext{
${ }^{4}$ We multiply the unnormalized CMB power spectrum by a constant, which is then adjusted to minimize $\chi^{2}$ with respect to the first year WMAP TT (up to $l=900$ ) and TE data (up to $l=512$ ) [44]. We assume the errors are uncorrelated.
}

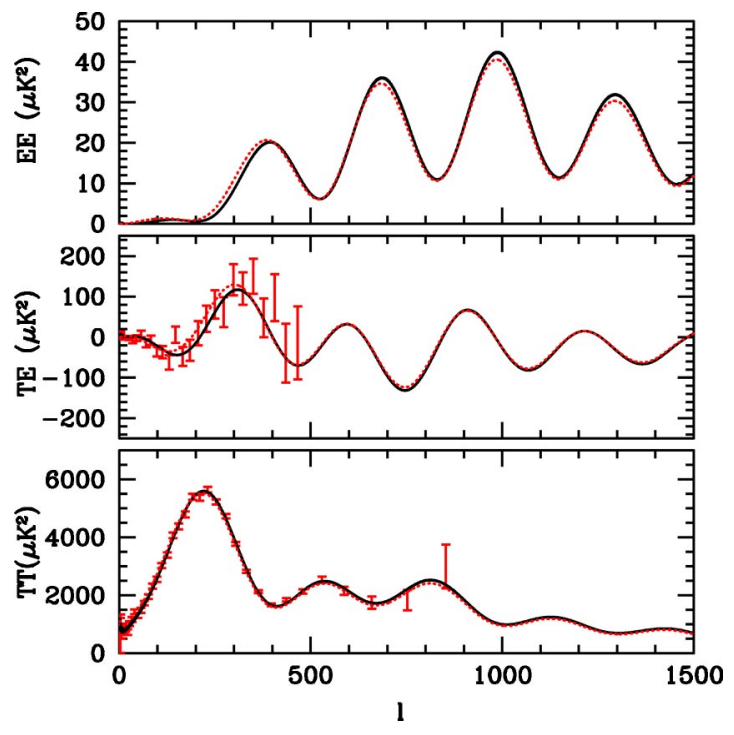

FIG. 5. The CMB temperature and polarization power spectrum $l(l+1) C_{l} /(2 \pi)$ for decaying particles with lifetimes greater than the age of the Universe. The data points with error bars are the binned data given by the WMAP team [44]. No particle decay (black solid line); long-lived particle decay with $\xi=2.4$ $\times 10^{-23} \mathrm{~s}^{-1}$, red dotted line; and $0.6 \times 10^{-23} \mathrm{~s}^{-1}$, (blue dashed line).

ferences in the TE and EE spectra. In Fig. 6 we plot the low$l$ results only; here the difference is more apparent. Since the overall normalization is increased, the low- $l$ multipoles of the high- $\tau$ models are raised. Current observations favor low power at large angular scales [1], so these models are not favored. In the TE data, the spectrum peaks at $l \sim 10$, which is again in contrast to the data, which is low at $l \sim 10$. The

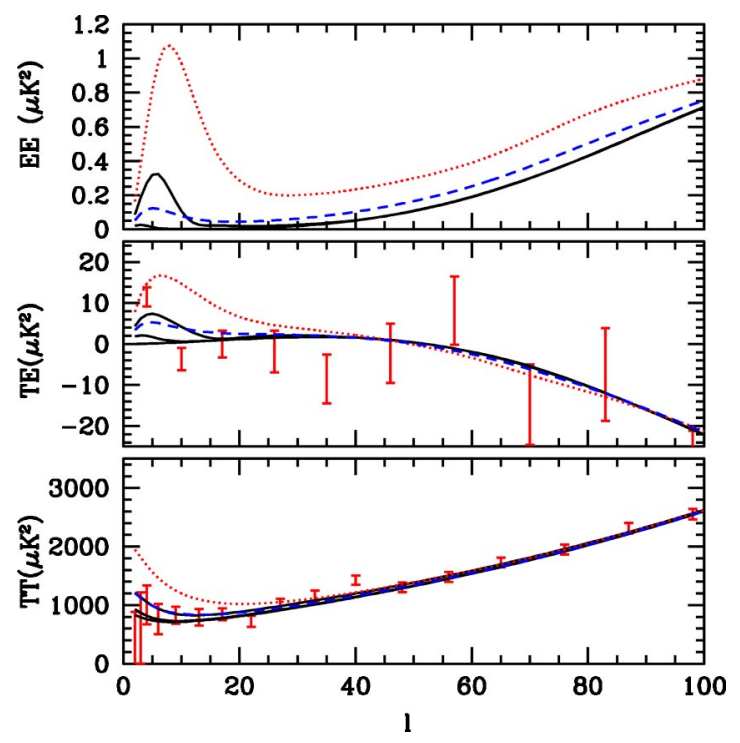

FIG. 6. Same as the previous figure, but for $l<100$ : $\xi=2.4$ $\times 10^{-23} \mathrm{~s}^{-1}$ (red dotted line) and $0.6 \times 10^{-23} \mathrm{~s}^{-1}$ (blue dashed line). We also plotted three curves for the no-particle-decay case (black solid line) which are almost indistinguishable except for the TE polarization; from top to bottom they are $\tau=0.17$, step-function reionization at $z<7$, and no reionization. 


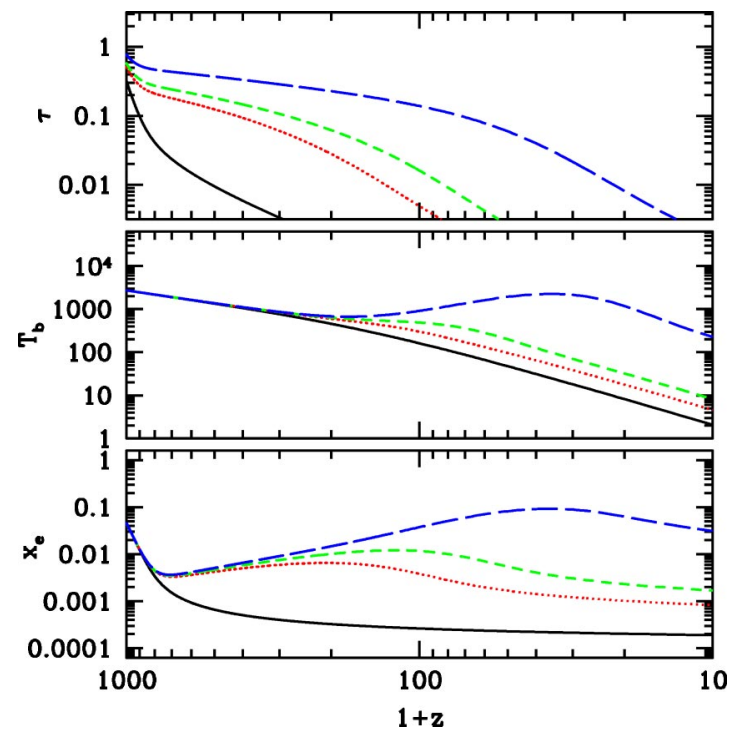

FIG. 7. The optical depth, IGM temperature, and ionization fraction for the standard no-reionization model (black solid line) and particle-decay-only models, all with $\chi=0.3$, and $\Gamma_{X}$ $=10^{-14} \mathrm{~s}^{-1}, \quad f_{X}\left(z_{\mathrm{eq}}\right)=0.5 \times 10^{-8} \quad($ red dotted line $) ; \Gamma_{X}=0.5$ $\times 10^{-14} \mathrm{~s}^{-1}, f_{X}\left(z_{\mathrm{eq}}\right)=10^{-8}$ (green short dashed line); and $\Gamma_{X}$ $=10^{-15} \mathrm{~s}^{-1}, f_{X}\left(z_{\mathrm{eq}}\right)=5 \times 10^{-8}$ (blue long dashed line).

greatest difference, however, is in the EE power spectrum, which should easily distinguish different models.

\section{B. Decaying particle with short lifetime}

If the primary particle has a lifetime less than the age of the Universe, its density will change dramatically when the age of the Universe is comparable to the lifetime. Moreover, its density today will be small. To be consistent with current observations of the CMB, which are well fit by a matter density comparable to that obtained from dynamical constraints in the present-day Universe, the cosmological density of the decaying particle must be small at the time of decoupling as well. Thus, $\Omega_{X}(z) \ll \Omega_{m}(z) \approx 1$. In this case,

$$
\frac{\Omega_{X}(z)}{\Omega_{b}(z)} \approx \frac{\Omega_{X 0}}{\Omega_{b 0}} e^{\Gamma_{X}\left[t_{0}-t(z)\right]}=\frac{n_{X \mathrm{eq}}}{n_{b \mathrm{eq}}} e^{-\Gamma_{X}\left[t(z)-t_{\mathrm{eq}}\right]},
$$

where $n_{X \text { eq }}, n_{b \text { eq }}$ are the number densities of the decaying particle and baryon at radiation matter equality, and the elapsed time is

$$
t(z)=\int_{z}^{\infty} \frac{d z}{(1+z) H(z)} \sim \frac{2}{3} \frac{1}{H_{0} \Omega_{0}^{1 / 2}} \frac{1}{(1+z)^{3 / 2}} .
$$

Unlike the long-decay-lifetime case, $\Gamma_{X}$ and $f_{X}$ must now be treated as independent parameters. We show a few examples of the ionization history for short-lifetime decaying particles in Figs. 7 and 8. The ionization rate is approximately constant until $t(z) \sim \Gamma_{x}^{-1}$, after which it decreases rapidly. In this scenario, the ionization fraction increases slowly after recombination, just as in the long-lifetime case. However, because the number density of the particle decreases, the ionization fraction peaks broadly at a certain

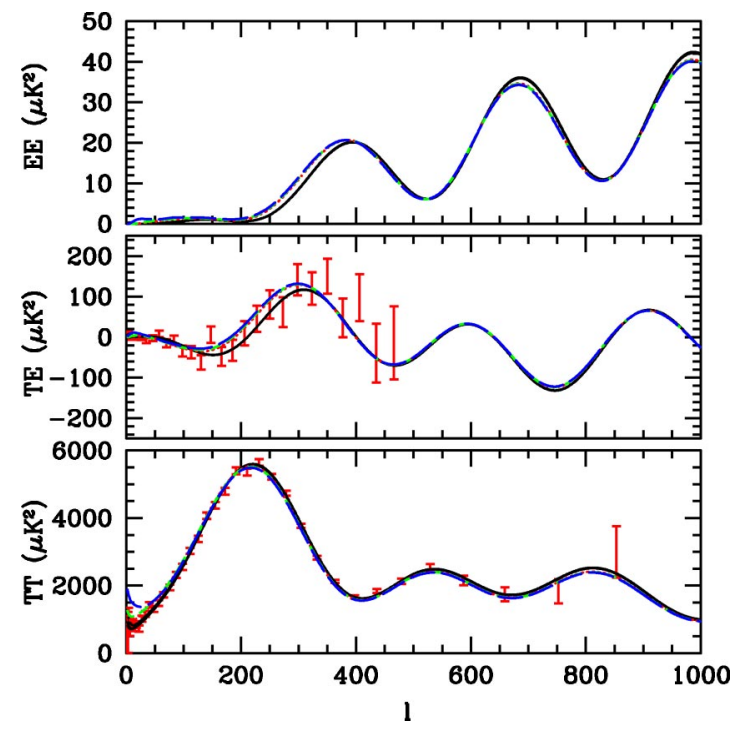

FIG. 8. The CMB temperature and polarization power spectrum. Same models as the previous figure.

redshift and then starts to decrease again. The peak position depends on the lifetime. The models plotted in Fig. 7 have $\Gamma_{X}^{-1}=10^{14} \mathrm{~s}, 2 \times 10^{15} \mathrm{~s}$, and $10^{15} \mathrm{~s}$, which correspond to the age of the Universe at $z=300,190$, and 65 respectively. Again, the ionization fraction can reach a few percent at $z$ $\sim 100$ without jeopardizing the structure of the CMB acoustic peaks. The temperature of the IGM departs from the CMB temperature at redshifts of a few hundred in these cases, but does not increase to a very high value because of the decreasing energy available for heating. The optical depth raises more sharply in this model, because there is more variation in the free-electron density at high redshift. As a result, the effect on the CMB is more apparent. We can see from Figs. 8 and 9 that the CMB temperature as well as polarization peaks have different shapes, especially apparent at high redshift. However, the TE correlation at low $l$ is less prominent for models with short lifetime, and even in the model with relatively long lifetime, the peak is shifted to greater $l$, in strong disagreement with the WMAP result. Inclusion of low-redshift reionization at $z=7$ results in only slight improvement. Based on this, particle decay with a short lifetime does not appear to help solve the high TE optical depth as observed by WMAP.

\section{Additional redshift dependence}

What if the decaying products are photons in the transparency window and deposit their energy differently? As we discussed in Sec. II, the effect of photons in the transparency window in the long-decay-lifetime case can be described with a suppression factor of $\tau_{S} \sim n(z) \sigma_{\text {eff }} c / H(z)$, which for baryons has a $(1+z)^{3 / 2}$ dependence.

Additional dependence on the redshift may also raise if the density of the decaying particle does not vary as (1 $+z)^{3} e^{-\Gamma_{X} t}$. For example, if the decaying particle is relativistic, its energy density decreases as $(1+z)^{4} e^{-\Gamma t^{\prime}}$, where $t^{\prime}$ is the proper time of the moving particle. Also, the decaying 


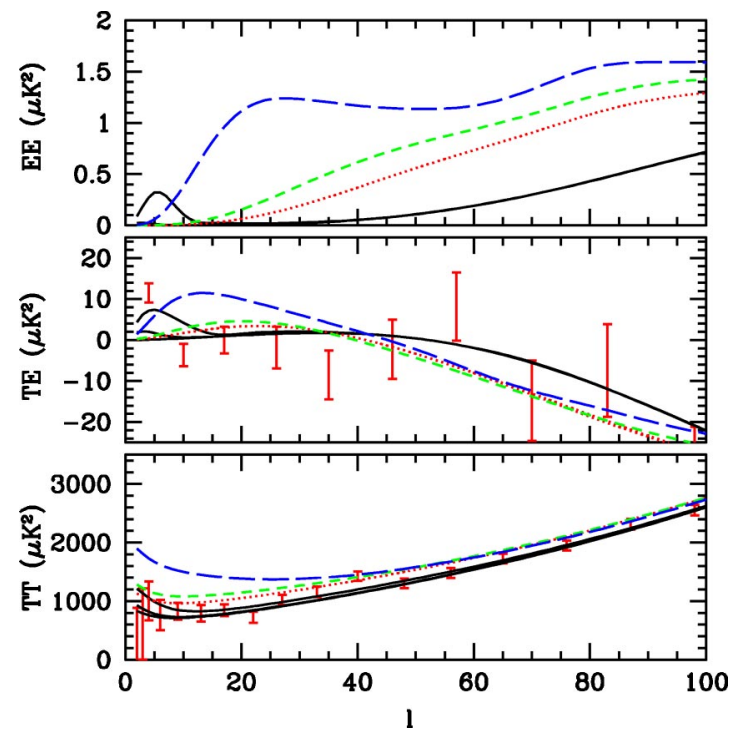

FIG. 9. Low- $l$ CMB temperature and polarization power spectrum. Same models as Fig. 8. The three black solid lines (almost indistinguishable except for the TE polarization) are, from top to bottom, for $\tau=0.17$, step function reionization at $z<7$, and no reionization.

particle could be continuously produced.

We now consider these effects on the CMB by multiplying $\xi$ with a factor $\tau_{S}(1+z)^{n}$, with $\tau_{S} \ll 1$. As an example, we consider models with $\tau_{S 100}=10^{-3}$ at $1+z=100$, and $n$ $=0,1.5,3$, and -3 . Obviously, at least for the $n=0$ case, the ionization induced by particle decay would be uninterestingly small if we still use the same parameters as in Sec. III A, since it is now suppressed by a factor of $\tau$. To see the effect of $z$ dependence, we increase $\xi$ by a factor of 1000 for the $n=0$ and 1.5 models, which cancels the small $\tau_{S}$ value we assumed. As it turns out, for the $n=3$ and -3 models this produces too large a deviation during the recombination era which could easily be ruled out, so for the $n=3$ and -3 models we increase $\xi$ by a factor of 100 . The ionization fraction, temperature, and optical depth are plotted in Fig. 10.

For the cases with $n>0$, the additional redshift dependence $(1+z)^{n}$ makes the ionization energy redshift away. This means that if we adopt parameters which do not spoil standard recombination, the effect on low redshift ionization must be very small. As shown in Fig. 11, this does not help explain the WMAP result.

From the above discussion, it seems that models with $n$ $<0$ may produce results which are more consistent with the WMAP data. For example, the $n=-3$ model tends to have relatively large effect at lower $l$. If, for example, the decaying particle is somehow associated with the dark energy which has a redshift dependence of $\rho \sim(1+z)^{0}$, then the $n=-3$ model might be realized.

Thus, we have investigated the ionization history and CMB for a variety of models. It appears long-lifetime models may help produce the large TE polarization at low $l$. Short life time models and models with additional redshift dependence typically work less well, except for particles or

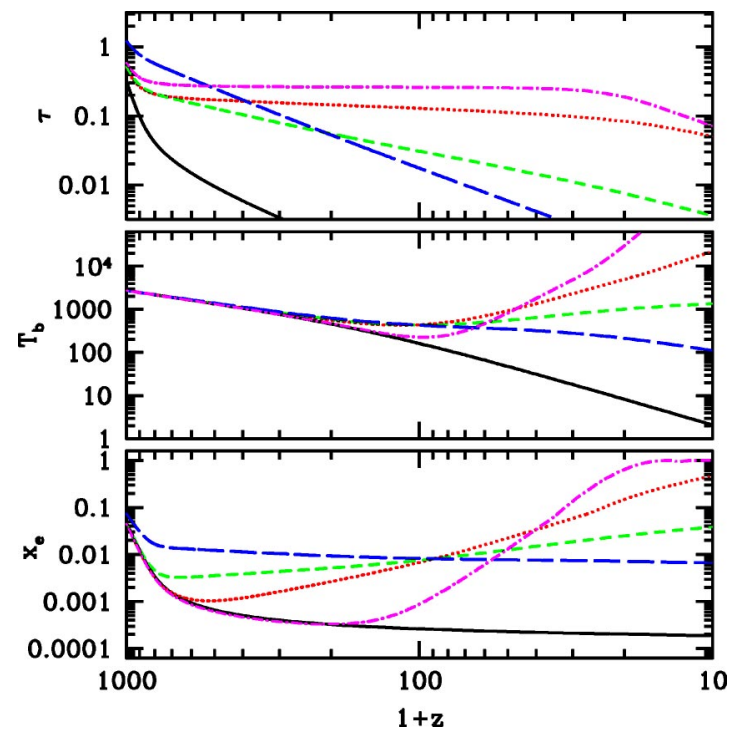

FIG. 10. The ionization fraction, temperature, and optical depth for models with photons in the transparency window or have nonstandard density evolution. The curves are standard no reionization model (black solid line) and particle decay only models, all with $\tau_{S 100}=10^{-3}, \quad \xi=0.6 \times 10^{-24}, \quad n=0 \quad$ (red dotted line); $\xi=0.6$ $\times 10^{-24}, n=1.5$ (green short dashed line); and $\xi=0.6 \times 10^{-25}, n$ $=3$ (blue long dashed line); $\xi=0.6 \times 10^{-25}, n=-3$ (magenta dashdotted line).

other energy sources whose density decrease slower than ordinary matter.

\section{OBSERVATIONAL CONSTRAINTS}

So far we have illustrated the effect of particles decay during the dark ages with several examples. We now use measurements of the CMB power spectra and diffuse backgrounds to place constraints to the decay-particle parameter space.

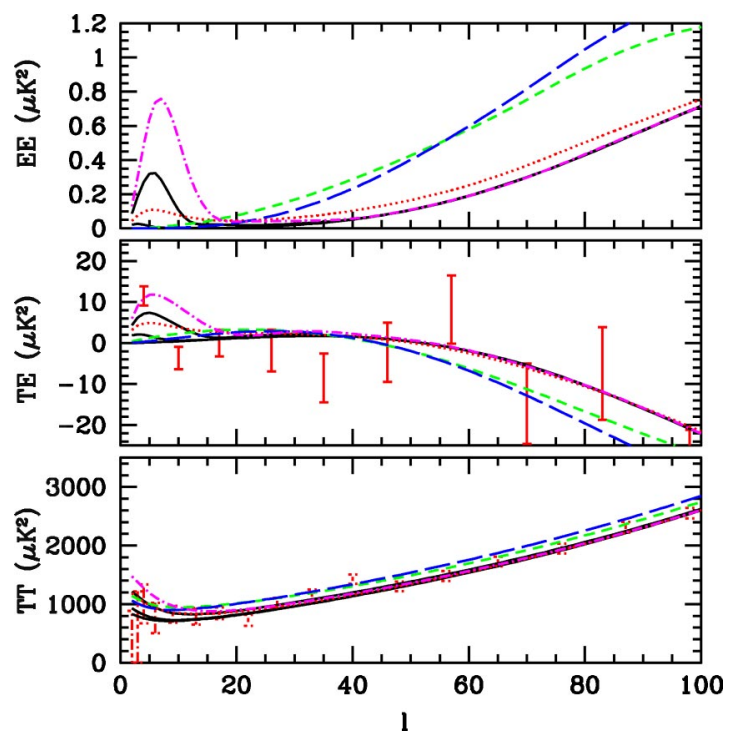

FIG. 11. The CMB temperature and polarization power spectrum for the models shown in Fig. 10. 


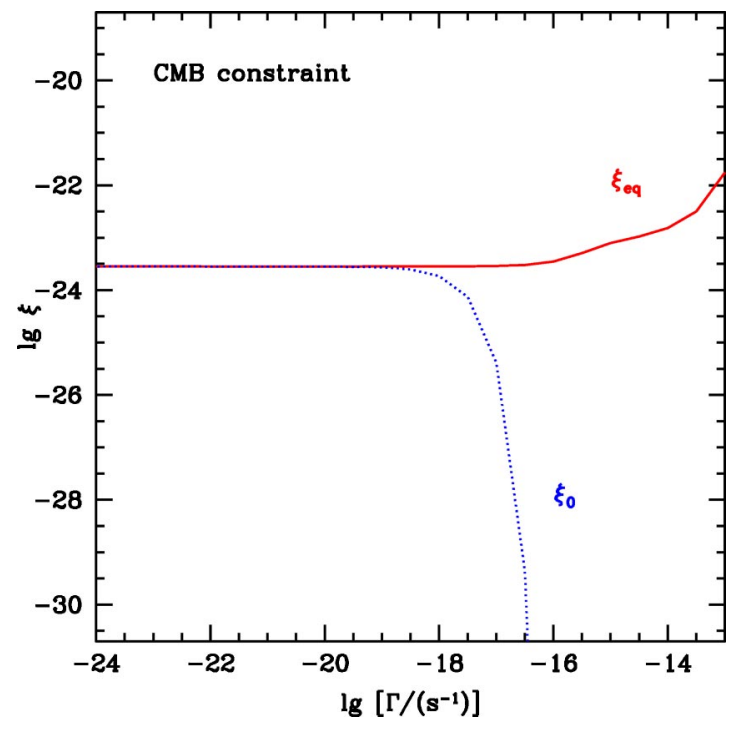

FIG. 12. WMAP $1 \sigma$ constraints on decaying particles. Plotted are $\xi \equiv \chi f_{X} \Gamma_{X}$, where $f_{X}=\Omega_{X} / \Omega_{b}$. The red solid curve shows constraint on the value at matter radiation equality $\xi_{\text {eq }}$; the blue dotted curve shows constraint on the value today $\xi_{0}$. Note that the WMAP constraint applies if the injected photon or electron energy does not fall in the transparency windows shown in Fig. 2 and Sec. II.

We first consider CMB constraints. We suppose that all of the decay energy is deposited instantly into the IGM, which then reionizes the Universe and changes the CMB power spectrum as described above. For each set of model parameters $\Gamma_{X}$ and $\xi$ we calculate $\chi^{2}$ for the WMAP TT and TE spectrum. We have $1403 \mathrm{TT}$ and TE data points. The $\chi^{2}$ distribution for 1403 degrees of freedom has a width of about 50 , so the $1 \sigma$ limit is defined by the line $\chi_{\min }^{2}+50$. The result is shown as the solid curve in Fig. 12; regions above the curve are ruled out. Note that these results do not apply if the particle decays to photons or electrons in the transparency window, as in these cases, the decay energy will not be deposited in the IGM but will instead propagate freely and appear in the diffuse radiation backgrounds.

In this case, the $\xi-\Gamma_{X}$ parameter space can be constrained from measurements of diffuse backgrounds $z=0$. The flux of decay radiation is then given by Eq. (5). with $z=0$. We then obtain bounds to the particle density and decay lifetime,

$$
N_{\gamma} \Gamma_{X} n_{X}(z)=\frac{4 \pi F(E)}{c} H(z)
$$

where $1+z=x M_{X} c^{2} / E$.

We now apply this result to the x-ray and $\gamma$-ray backgrounds. The observed cosmic x-ray background (in units of $\mathrm{cm}^{-2} \mathrm{~s}^{-1} \mathrm{sr}^{-1}$ ) can be modeled as [30]

$$
F_{X}=\left\{\begin{array}{rr}
8\left(\frac{E}{\mathrm{keV}}\right)^{-0.4}, & 0.2 \mathrm{keV}<E<25 \mathrm{keV} \\
380\left(\frac{E}{\mathrm{keV}}\right)^{-1.6}, & 25 \mathrm{keV}<E<350 \mathrm{keV}, \\
2\left(\frac{E}{\mathrm{keV}}\right)^{-0.7}, & 350 \mathrm{keV}<E<2 \mathrm{MeV}
\end{array}\right.
$$

The $\gamma$-ray background was measured by the Energetic Gamma Ray Experiment Telescope (EGRET). At about 100 $\mathrm{MeV}$, the flux is $1.45 \times 10^{-5} \mathrm{~cm}^{-2} \mathrm{~s}^{-1} \mathrm{sr}^{-1}$. A fit for the whole energy range of $30 \mathrm{MeV}-100 \mathrm{GeV}$ was [47]

$$
F_{\gamma}=1.73 \times 10^{-5}\left(\frac{E}{100 \mathrm{MeV}}\right)^{-2.11} \mathrm{~cm}^{-2} \mathrm{~s}^{-1} \mathrm{sr}^{-1}
$$

More recent re-analysis found a greater galactic background, and therefore the extra-galactic background is lowered $[48,49]$. Of course, distant quasars and stars also contribute to this background, so here we use the measured background Eq. (32) as a conservative upper limit.

At each photon energy $E$, given the background flux $F(E)$, we can derive a bound on $n_{\text {eq }}$ for each decay photon energy $x M_{X} c^{2}$ by applying Eq. (30) to the flux (with the restriction that the maximum redshift to be less than $z$ $\sim 1000$ ). We run through the energy range of $1 \mathrm{keV}-100 \mathrm{GeV}$, at each energy using the flux limits given in Eqs. (30) and (32) to derive a bound, and look for the most restrictive constraint on $n_{e} q$ for all energies. As expected, except for short-lived particles, the constraint comes mainly from emission at $z=0$. The results for decay photon in the transparency window $\quad x M_{X} c^{2}=100 \mathrm{keV}, 1 \mathrm{MeV}$, $10 \mathrm{MeV}, 100 \mathrm{MeV}, 1 \mathrm{GeV}, 10 \mathrm{GeV}, 100 \mathrm{GeV}$, are plotted as $\xi_{\text {eq }}$ in Fig. 13. On the same figure we also plot the corresponding values of today, $\xi_{0}$. In terms of the decaying particle at radiation-matter equality, particles with short lifetimes are less constrained than the ones with long lifetimes. However, we do not expect any of these short-lifetime particles to remain today, as shown in Fig. 13.

Depending on the energy of the observed photon, the diffuse $\mathrm{x}$-ray and $\gamma$-ray background constraints are generally more stringent than the CMB constraint except for shortlived particles. One must remember, however, that the CMB constraint applies only outside the transparency window, whereas the $\gamma$-ray constraint applies in the transparency window.

\section{CONCLUSIONS}

In this paper we have investigated particle decay during the dark ages. Such particle decay could induce partial ionization of the Universe, and thus provide a potential alternative to early star formation as an explanation for the WMAP TE measurement. 


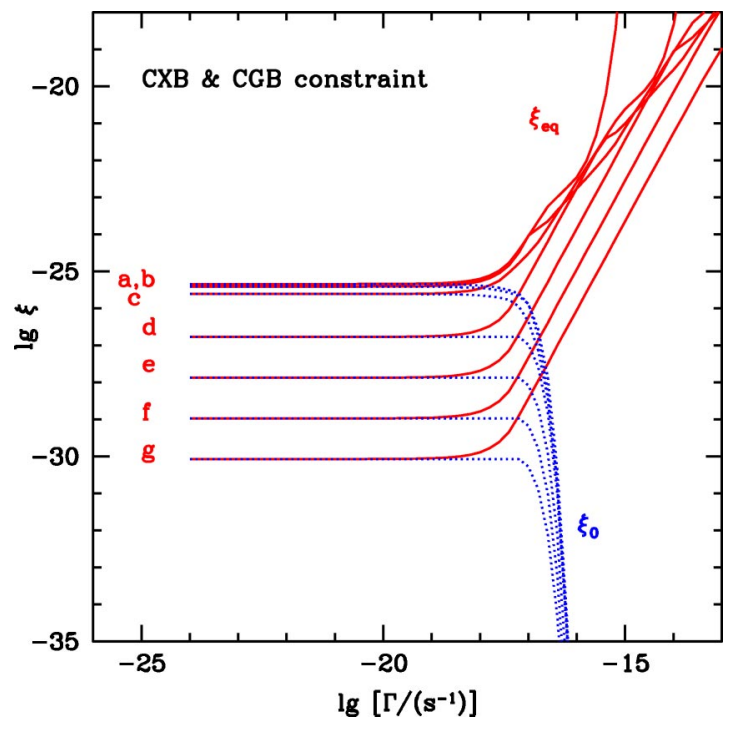

FIG. 13. Constraint of $\xi$ based on diffuse x-ray and $\gamma$-ray background. The red solid curve shows constraint on the value at matter radiation equality $\xi_{\text {eq }}$; the blue dotted curve shows constraint on the value today $\xi_{0}$. The curves are for photon energy (a) $100 \mathrm{keV}$, (b) 1 $\mathrm{MeV}$, (c) $10 \mathrm{MeV}$, (d) $100 \mathrm{MeV}$, (e) $1 \mathrm{GeV}$, (f) $10 \mathrm{GeV}$, (g) 100 $\mathrm{GeV}$. Note that the $\mathrm{x}$-ray and $\gamma$-ray constraints do not apply for photon and electron injection energies that fall outside the transparency windows.

We considered how the decay energy is converted to ionization energy. We conclude that in many cases, a shower of electrons and $\mathrm{x}$-ray photons are produced, in which case a sizable fraction $(0.1-0.3)$ of the energy can be converted to ionization energy in situ, with comparable amount of energy going into heating the gas. However, there are important exceptions. Photons in the energy range $100 \mathrm{keV}-1 \mathrm{TeV}$ can escape, carrying with them most of the energy. Electrons in the energy range $1 \mathrm{GeV}-50 \mathrm{TeV}$ lose most of their energy by inverse-Compton scattering $\mathrm{CMB}$ photons into the above energy range. In these cases the ionization energy is deposited over a range of redshifts, and the energy deposition is proportional to $\tau_{S}=n(z) \sigma_{\text {eff }} c / H(z)$, where $n(z)$ is the density of target particles, which can be baryons or the CMB photons. Because of the small value of the optical depth $\tau_{S}$, the decay rate must be very large to affect ionization. We can study reionization in these models by considering additional $1+z$ dependence. In most cases, though, the models will be ruled out, as seen in Fig. 13, by diffuse backgrounds.

The extra energy input from particle decays can be parameterized by the ionization energy input parameter $\xi$ $=\chi f_{X} \Gamma_{X}\left(\xi\right.$ has units of $\left.\mathrm{s}^{-1}\right)$, where $\chi$ is the efficiency, $f_{X}=\Omega_{X} / \Omega_{b}$, and $\Gamma_{X}$ the decay rate. If the lifetime of the decaying particle is longer than the age of the Universe, the situation is particularly simple since the result depends entirely on $\xi$. For short-lived particles, one must specify both $\Gamma_{X}$ and $\xi$. We studied the ionization history and CMB temperature and polarization anisotropy for different cases. Although particle decays could partially ionize the Universe at high redshift and produce a high optical depth, we found that in most cases they do not reproduce the WMAP result very well. The TE polarization does not peak at $l \sim 2$ but at $l$ $\sim 10$, for example. We should pointed out however, this is not a unique problem with particle-decay induced reionization, but is also seen in other models with extended partial reionization history. We also found that the EE spectrum is a sensitive probe to the ionization history. Furthermore, if reionization occurs at high redshift, there is a change in the shape and position of the acoustic peaks. The ionization history is affected if the extra energy input has additional dependence on the redshift. Typically, for an additional redshift dependence of $(1+z)^{n}$ with $n>0$, the fit to CMB data is not improved, because the extra energy input at early times will spoil recombination. Models with $n<0$ may be helpful, but some exotic mechanism is needed for generating such a redshift dependence.

We have obtained constraints on particle decays during the dark ages using the WMAP data as shown in Fig. 12. We found $\xi<10^{-24} \mathrm{~s}^{-1}$ for the long-lifetime case, and a slightly weaker bound for the short-lifetime case. However, the short-lived particles decay at high redshift and we do not expect to see any left today. We also obtained constraints on the decaying particle from the observed diffuse $\mathrm{x}$-ray and $\gamma$-ray backgrounds. This constraint is generally more stringent than the CMB constraint, but it actually applies to a different situation; i.e. the decay products are mainly photons in the energy range of the transparency window, where they can propagate freely across the Universe and contribute very little energy to ionization.

The extra energy input also heats up the IGM during the dark age, and the temperature can rise to $10^{3-4} \mathrm{~K}$. InverseCompton scattering of free electrons can induce distortion in the CMB blackbody spectrum, but the effect is unobservably small $\left(y<10^{-8}\right)$.

If the dark-matter particle can decay, it may affect the estimation of cosmological parameters. To see how each parameter is affected, we can calculate the correlation matrix, which is related to the covariance matrix [50] by

$$
\mathbf{r}_{i j}=\mathbf{C}_{i j} / \sqrt{\mathbf{C}_{i i} \mathbf{C}_{j j}}
$$

where the covariance matrix is given by the inverse of the Fisher matrix $\mathbf{C}=\mathbf{F}^{-1}$, with

$$
\mathbf{F}_{i j}=\sum_{l}\left[\frac{1}{\sigma_{C_{l}^{T T}}^{2}} \frac{\partial C_{l}^{T T}}{\partial \theta_{i}} \frac{\partial C_{l}^{T T}}{\partial \theta_{j}}+\frac{1}{\sigma_{C_{l}^{T E}}^{2}} \frac{\partial C_{l}^{T E}}{\partial \theta_{i}} \frac{\partial C_{l}^{T E}}{\partial \theta_{j}}\right],
$$

and $\theta_{i}$ are the cosmological parameters to be estimated. We plot $r_{i j}^{2}$ in Fig. 14.

In making Fig. 14, we have taken a fiducial model with the WMAP best fits with the exception $\tau=0.037$ which corresponds to a sudden reionization with $z_{r e i}=6.0$. Choosing different fiducial models may affect the error estimates slightly. In addition to the standard parameters (physical density of baryons $\Omega_{b} h^{2}$, cold dark matter $\Omega_{c} h^{2}$, Hubble con- 

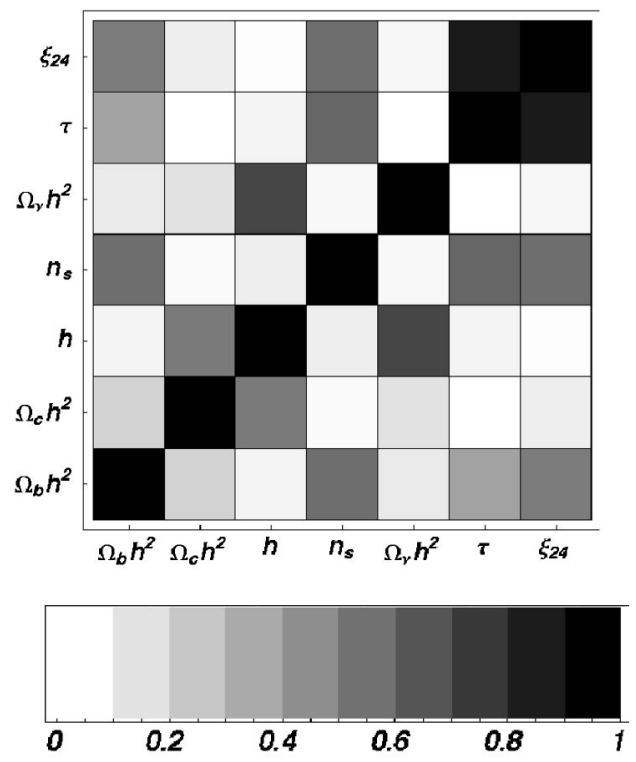

FIG. 14. The (squared) correlation matrix for cosmological parameters. The parameters are the physical density of baryons $\Omega_{b} h^{2}$, cold dark matter $\Omega_{c} h^{2}$, Hubble constant $h$, power index for primordial density perturbation $n_{s}$, neutrino density $\Omega_{v} h^{2}$, Thomson optical depth (see text) $\tau$, and the extra ionization parameter $\xi_{24}$ $\equiv 10^{24} \xi$. We assume a flat Universe.

stant $h$, power index for primordial density perturbation $n_{s}$, neutrino density $\Omega_{\nu} h^{2}$, Thomson optical depth $\tau$ ), we have added the extra ionization input energy $\xi_{24} \equiv 10^{24} \xi$ in the long-lived decaying-particle case. We will not consider the short-live case since it is much more model dependent. As expected, $\xi$ correlates strongly with the Thomson optical depth due to low-redshift stellar light; it will thus be difficult to distinguish them from CMB observations alone. Both $\tau$ and $\xi$ correlates strongly with baryon density $\Omega_{b} h^{2}$ and the primordial spectral index $n_{s}$. If a decaying particle exists but is neglected in the fit, then results for the values of other cosmological parameters may be biased, and the error bars may be underestimated. In Fig. 15 we plot error ellipses for $\Omega_{b} h^{2}-\xi$ and $n_{s}-\xi$ after marginalizing over the other parameters.

There are other ways that particle decays during the cosmic dark ages could play a role in cosmology. Decays might affect the recombination process [28]. Particle decays could produce a surfeit of free electrons after recombination; these extra electrons could then facilitate the formation of $\mathrm{H}_{2}$ molecules and thus potentially enhance the star-formation rate. On the other hand, particle decay may also heat up the gas, thus increasing the Jeans mass of the primordial gas and suppressing early star formation. The final outcome requires detailed investigation which is beyond the scope of the present paper. Finally, if the contribution of the particle to reionization is significant, it may not require formation of structure at high redshift, thus eliminating one objection to warm dark-matter models.
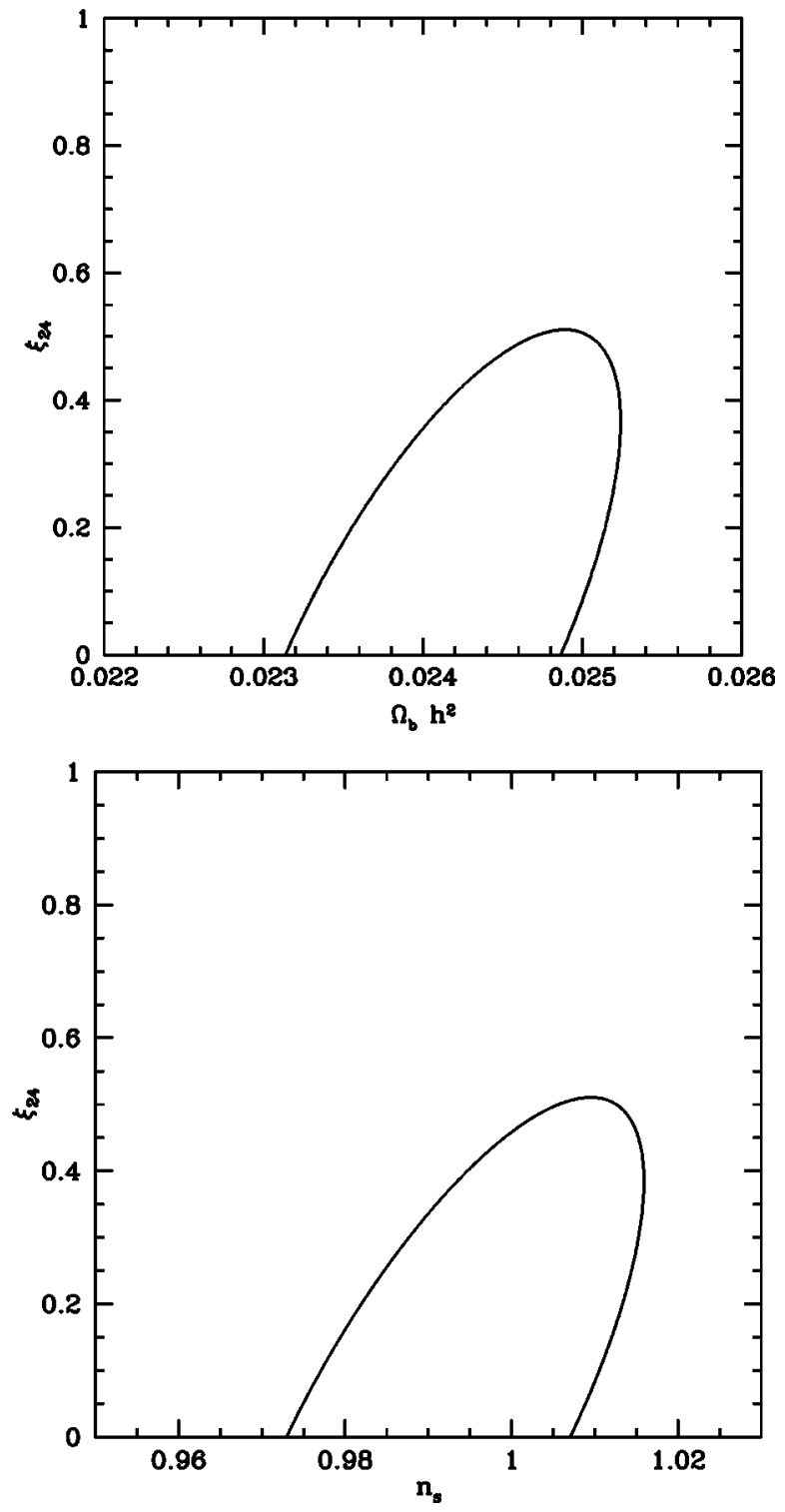

FIG. 15. The error ellipses for $\Omega_{b} h^{2}$ vs $\xi_{24}$ and $n_{s}$ and $\xi_{24}$. $\xi$ is positive from its physical interpretation.

With future experiments, we expect to obtain more precise information on the ionization history than we have now, and should a decaying particle with $t>10^{13} \mathrm{~s}$ exist, we may discover it through indirect observations such as those discussed here.

\section{ACKNOWLEDGMENT}

We thank R. Sunyaev, E. Scannapieco, S.-P. Oh, and M. Kaplinghat for helpful comments and discussions. The CAMB code we used in this research was developed by A. Lewis and A. Challinor. X.C. is supported by NSF grant PHY9907949, and M.K. is supported by NASA grant NAG 5-9821 and DOE grant DE-FG 03-92-ER40701. 
[1] A. Kogut et al., Astrophys. J., Suppl. Ser. 148, 161 (2003); D.N. Spergel et al., ibid. 148, 175 (2003).

[2] M. Zaldarriaga, Phys. Rev. D 55, 1822 (1997); M. Kaplinghat et al., Astrophys. J. 583, 24 (2003); G.P. Holder et al., ibid. 595, 13 (2003).

[3] M. Fukugita and M. Kawasaki, Mon. Not. R. Astron. Soc. 343, L25 (2003); R. Cen, Astrophys. J. Lett. 591, L5 (2003); N. Yoshida et al., Astrophys. J. 592, 645 (2003); A. Sokasian et al., astro-ph/0307451; Z. Haiman and G.P. Holder, Astrophys. J. 595, 1 (2003).

[4] N.Y. Gnedin and J.P. Ostriker, Astrophys. J. 486, 581 (1997); Z. Haiman and A. Loeb, ibid. 483, 21 (1997); 503, 505 (1998); Astrophys. J. Lett. 521, L9 (1999); W.A. Chiu and J.P. Ostriker, Astrophys. J. 534, 507 (2000); N.Y. Gnedin, Astrophys. J. Lett. 535, L75 (2000); astro-ph/0110290; R. Cen and P. MacDonald, Astrophys. J. 570, 457 (2002).

[5] X. Fan et al., Astron. J. 123, 1247 (2002); T. Theuns et al., Astrophys. J. Lett. 567, L103 (2002); L. Hui and Z. Haiman, Astrophys. J. 596, 9 (2003).

[6] R. Cen, Astrophys. J. 591, 12 (2003); S. Wyithe and A. Loeb, ibid. 586, 693 (2003).

[7] X. Chen et al., Mon. Not. R. Astron. Soc. 346, L31 (2003).

[8] Y. Rephaeli and A.S. Szalay, Phys. Lett. 106B, 73 (1981); D.W. Sciama, Mon. Not. R. Astron. Soc. 198, 1S (1982); Phys. Rev. Lett. 65, 2839 (1990); J.A. Adams, S. Sarkar, and D.W. Sciama, Mon. Not. R. Astron. Soc. 301, 210 (1998); Modern Cosmology and the Dark Matter Problem (Cambridge University Press, Cambridge, England, 1993).

[9] M.S. Turner, G. Steigman, and L.M. Krauss, Phys. Rev. Lett. 52, 2090 (1984); G. Gelmini, D.N. Schramm, and J.W.F. Valle, Phys. Lett. 146B, 311 (1984); A.G. Doroshkevich and M.Yu. Khlopov, Yad. Fiz. 39, 869 (1984) [Sov. J. Nucl. Phys. 39, 551 (1984)]; Mon. Not. R. Astron. Soc. 211, 277 (1984); A.G. Doroshkevich, A.A. Klypin, and M.Yu. Khlopov, ibid. 239, 923 (1989); Z.G. Berezhiani and M.Yu. Khlopov, Z. Phys. C 49, 73 (1991); Yad. Fiz. 52, 96 (1990) [Sov. J. Nucl. Phys. 52, 60 (1990)].

[10] S. Dodelson and J.M. Jubas, Mon. Not. R. Astron. Soc. 266, 886 (1994).

[11] S. Bowyer et al., Astrophys. J. 526, 10 (1999).

[12] N. Cabibbo, G.R. Farrar, and L. Maiani, Phys. Lett. 105B, 155 (1981); P. Salati and J.C. Wallet, ibid. 144B, 61 (1984).

[13] J. Ellis, J. Lopez, and D.V. Nanopoulos, Phys. Lett. B 247, 257 (1990).

[14] V. Berezinsky, A. Masiero, and J.W.F. Valle, Phys. Lett. B 266, 382 (1991).

[15] T. Asaka et al., Phys. Rev. D 58, 023507 (1998).

[16] D.J.H. Chung, E.W. Kolb, and A. Riotto, Phys. Rev. D 59, 023501 (1999).

[17] A.G. Doroshkevich and P.D. Naselsky, Phys. Rev. D 65, 123517 (2002).

[18] H.B. Kim and J.E. Kim, Phys. Lett. B 527, 18 (2002).

[19] S. Hansen and Z. Haiman, Astrophys. J. 600, 26 (2004).

[20] J.L. Feng, A. Rajaraman, and F. Takayama, Phys. Rev. Lett.
91, 011302 (2003); Phys. Rev. D 68, 063504 (2003).

[21] X. Bi, M. Li, and X. Zhang, Phys. Rev. D (to be published), hep-ph/0308218.

[22] P. He and L. Fang, Astrophys. J. Lett. 568, L1 (2002).

[23] R. Cen, Astrophys. J. Lett. 546, L77 (2001).

[24] R. Cen, Astrophys. J. Lett. 549, L195 (2001).

[25] F.J. Sanchez-Salcedo, Astrophys. J. Lett. 591, L107 (2003).

[26] P.H. Frampton and S.L. Glashow, Phys. Rev. Lett. 44, 1481 (1980); J. Ellis, T.K. Gaisser, and G. Steigman, Nucl. Phys. B177, 427 (1981); V. Berezinsky, M. Kachelriess, and A. Vilenkin, Phys. Rev. Lett. 79, 4302 (1997); M. Birkel and S. Sarkar, Astropart. Phys. 9, 297 (1998).

[27] P.J.E. Peebles, S. Seager, and W. Hu, Astrophys. J. Lett. 539, L1 (2000).

[28] R. Bean, A. Melchiorri, and J. Silk, Phys. Rev. D 68, 083501 (2004).

[29] See, e.g., M. Kamionkowski, in The Gamma-Ray Sky with Compton GRO and SIGMA, edited by M. Signore, P. Salati, and G. Vedrenne (Kluwer Academic, Dordrecht, 1995), p. 113-134, astro-ph/9404079.

[30] S. Kasuya, M. Kawasaki, and N. Sugiyama, Phys. Rev. D 69, 023512 (2004).

[31] E. Pierpaoli, Phys. Rev. Lett. 92, 031301 (2004).

[32] A.A. Zdziarski and R. Svensson, Astrophys. J. 344, 551 (1989).

[33] R. Sunyaev and E.M. Churazov, Astron. Lett. 22, 648 (1996).

[34] A.A. Zdziarski, Astrophys. J. 335, 786 (1988).

[35] A.A. Zdziarski and R. Svensson, Astrophys. J. 349, 415 (1990).

[36] M. Kawasaki and T. Moroi, Astrophys. J. 452, 506 (1995).

[37] R.J. Protheroe, T. Stanev, and V.S. Berezinsky, Phys. Rev. D 51, 4134 (1995).

[38] P. Gondolo, Phys. Lett. B 295, 104 (1992).

[39] K. R. Lang, Astrophysical Formulae, 3rd ed. (Springer, Berlin, 1999).

[40] J.M. Shull and M.E. van Steenberg, Astrophys. J. 298, 268 (1985).

[41] J. Miralda-Escudé, M. Haehnelt, and M.J. Rees, Astrophys. J. 530, 1 (2000).

[42] S. Seager, D.D. Sasselov, and D. Scott, Astrophys. J. 523, 1 (1999).

[43] http://camb.info.

[44] http://lambda.gsfc.nasa.gov.

[45] D.J. Fixsen et al., Astrophys. J. 473, 576 (1996).

[46] W.A. Chiu, X. Fan, and J.P. Ostriker, Astrophys. J. 599, 759 (2003).

[47] P. Sreekumar et al., Astrophys. J. 494, 523 (1998).

[48] A.W. Strong, I.V. Moskalenko, and O. Reimer, astro-ph/0306345.

[49] U. Keshet, E. Waxman, and A. Loeb, J. Cosmol. Astropart. Phys. 04, 006 (2004).

[50] G. Jungman, M. Kamionkowski, A. Kosowsky, and D.N. Spergel, Phys. Rev. Lett. 76, 1007 (1996); Phys. Rev. D 54, 1332 (1996). 